\title{
TROCA VELOCIDADE-ACURÁCIA EM TAREFA DE CONTORNAR FIGURAS GEOMÉTRICAS
}

\author{
DR. VICTOR HUGO ALVES OKAZAKI \\ Doutor em Biodinâmica do Movimento Humano pela USP e \\ Professor Adjunto da Universidade Estadual de Londrina (Paraná - Brasil) \\ e-mail: vhaokazaki@gmail.com \\ Site: http://okazaki.webs.com \\ MS. DANIELLE BRANDALIZE \\ Mestre em Educação Física pela UFPR (Paraná - Brasil) \\ e-mail: danibranda@yahoo.com.br \\ MS. NATALIA KOPP OKAZAKI \\ Mestre em Educação Física pela UFPR (Paraná - Brasil) \\ e-mail: nataliakopp@hotmail.com \\ MS. BIANCA DRABOVSKI \\ Mestre em Educação Física pela UFPR (Paraná - Brasil) \\ e-mail: biancadrabovski@hotmail.com

\section{DR. IVERSON LADEWIG} \\ Doutor em Desenvolvimento Motor e Estudos Esportivos pela \\ Universidade de Pittsburgh e Professor Associado do Depto. de \\ Educação Física da Universidade Federal do Paraná (Paraná - Brasil) \\ e-mail: iverson@ufpr.br
}

\section{RESUMO}

O presente estudo analisou o paradigma da relação inversa velocidade-acurácia, através da lei de Fitts, em tarefas de contornar figuras geométricas. Um software especialmente desenvolvido permitiu manipular a forma (quadrado, triângulo e diamante) e o tamanho da figura (perímetro e número de voltas para contorná-la), e a espessura da linha, fornecendo índices de dificuldade (ID) entre 2,32 e 8,46 bits. Foi verificada a robustez da lei de Fitts na tarefa de contornar figuras geométricas. Ademais, foi sugerido que, em análises que contemplem o número de repetição da tarefa (por exemplo: com movimentos discretos e cíclicos), o índice de dificuldade pode ser calculado a partir do número de tentativas, apresentando uma relação TM $\times$ ID exponencial ao invés de linear.

PALAVRAS-CHAVE: Velocidade; precisão; movimento; controle motor. 


\section{INTRODUÇÃO}

Um dos princípios mais fundamentais do movimento humano é o que trata da relação inversa na geração da velocidade e no controle da acurácia de um movimento (SPARROW; SPARROW, I99I; BOOTSMA et al., 2004). Este princípio estabelece que a maior exigência na acurácia, tende a ser compensada pela redução na velocidade do movimento. Ao passo que, quando essa demanda na precisão é reduzida há maior possibilidade na geração de velocidade no movimento (WOODWORTH, 1899; FITTS, 1954; FITTS; PETERSEN, 1964). Woodworth (1899) foi o primeiro pesquisador a tentar identificar as variáveis responsáveis pela relação entre a velocidade e a acurácia para tentar descobrir os processos subjacentes ao seu controle. Através da tarefa de realizar traços em direção a duas linhas paralelas, foi demonstrado que o aumento na velocidade e a oclusão do feedback visual resultaram em maior erro de resposta. Assim, Woodworth ( 1899 ) apontou que a menor possibilidade de utilização de feedback, para as correções necessárias que garantam a acurácia no movimento, seria a principal responsável pela relação inversa velocidadeacurácia. Suporte para a explicação da relação inversa velocidade-acurácia através da utilização do feedback também foi verificada em estudos posteriores (CROSSMAN; GOODEVE, 1963/I983; BEGGS; HOWART, 1972; MEYER et al., 1982, 1988). Entretanto, foi a partir da explicação fornecida por Paul Fitts (FITTS, 1954; FITTS; PETERSEN, 1964), que conseguiu expressar matematicamente esta relação inversa, que este paradigma ficou mais conhecido.

Utilizando a teoria da informação (SHANNON, 1948; MILLER, 1953), foi levantada a hipótese de que a capacidade de transmissão da informação fixa do sistema motor possibilita um caminho para analisar a relação entre a distância (D), o tamanho do alvo (A) e o tempo de movimento (TM). Por conseguinte, a dificuldade da tarefa poderia ser mensurada em bits utilizando uma informação métrica e que, considerando uma tarefa de movimento, a informação seria transmitida através de um canal de comunicação estocástico de ruído que modela o comportamento do sistema motor humano (FITTS, 1954). Para tanto, foi utilizada uma adaptação do teorema 17 de Shannon (1948), que expressou a capacidade de informação efetiva $C$ (em bitts/segundo), de um canal com comprimento de banda $B(\mathrm{em} \mathrm{Hz})$, tal como: $C=B \log _{2}((S+N) / N)$, no qual $N$ é a potência do ruído e $S$ é a potência do ruído. Fitts afirmou que, em taxa máxima de transmissão da informação (por exemplo, realizar um movimento com maior velocidade possível), o sistema motor humano se comporta de acordo com uma relação logarítmica através da seguinte 
adaptação no teorema I/TM com B, 2D com $S+N$, e A com N para obter a relação de troca velocidade-acurácia proposta na equação: $M T=a+b \log _{2}(2 D / A)$, no qual $a$ e $b$ são constantes empíricas, e o logaritmo de 2D/A foi chamado de índice de dificuldade (ID).

Fitts (1954) proporcionou suporte para sua hipótese através de três experimentos que analisaram tarefas, tais como: toques repetitivos com uma ponteira, transferência de discos e transferência de pinos. Foi demonstrado que o tempo de movimento aumentou proporcionalmente com o acréscimo no índice de dificuldade (ID), através do aumento na distância do movimento ou da diminuição do tamanho do alvo. Assim, quanto maior a demanda de acurácia na tarefa, menor a velocidade no movimento (FITTS, 1954). Devido à consistência do paradigma a formulação de Fitts ganhou status de lei, recebendo suporte de diferentes tarefas, tais como: movimentos discretos (FITTS; PETERSON, 1964; SLIFKIN; GRILLI, 2006; OKAZAKI et al., 2008; HUYS et al., 20 I 0), movimentos cíclicos (FITTS, 1954; GUIARD, I997; WU et al., 20 I0), movimentos de agarrar (BOOTSMA et al., 1994), movimentos de flexão de punho (CROSSMAN; GOODEVE, 1963/I 983; MEYER et al., 1982), movimentos de extensão de braço (KERR; LANGOLF, 1977), movimentos feitos com a cabeça (ANDRES; HARTUNG, 1989) e movimentos bimanuais (MOTTET et al., 200 I). Contudo, os estudos que analisaram a lei de Fitts, em sua maioria, foram limitados a tarefas laboratoriais simples, unidimensionais, com controle predominantemente via feedfoward, além de possuírem pouca validade ecológica.

Neste ínterim, a tarefa de contornar figuras geométricas, utilizando-se o mouse em computador, permite a manipulação das mesmas variáveis controladas por Fitts, tais como distância do movimento, através do tamanho no perímetro da figura, e tamanho do alvo, por meio da espessura da linha da figura. Por conseguinte, é possível a análise da robustez da lei de Fitts em tarefas motoras bidimensionais de restrição espacial. Em particular, tal tarefa também permite a análise de diferentes mudanças de direção (restrição espacial bidimensional) por meio da manipulação nas formas das figuras utilizadas. Por exemplo, um triângulo equilátero possui ângulos mais agudos em seus vértices comparados aos ângulos nos vértices de um quadrado. Assim, seriam esperadas maiores restrições espaciais em figuras com ângulos que demandem maior mudança de direção, pois necessitam de maior controle na precisão por meio da regulação das forças de aceleração e desaceleração no movimento (FIUZA; OKAZAKI, 2009). Além disso, estas tarefas de manipulação, com o uso mouse em computador, também já fazem parte do cotidiano de grande parte das pessoas. Outra particularidade é o fato de que, na tarefa de contornar 
figuras geométricas, em função do tempo para completar a tarefa e das restrições espaciais impostas, há maior possibilidade para a utilização do feedback durante seu desempenho. Esta característica não tem sido contemplada nos estudos que analisaram a lei de Fitts. Por conseguinte, a análise da robustez da lei de Fitts em tarefa de contornar figuras geométricas em computador tem potencial para o entendimento de classes de habilidades antes não investigadas no paradigma. Ademais, nas tarefas que analisaram o paradigma da relação inversa velocidade-acurácia, o ID foi analisado apenas em função das restrições da distância e do tamanho do alvo, não sendo considerados os números de repetições realizadas nas tarefas (FITTS; PETERSEN, 1964; GUIARD, 1997).

Como o maior número de repetições num movimento permite maior tempo para a realização da tarefa, maior possibilidade para utilização do feedback (ou feedfoward) sensorial também é proporcionada. Assim, o movimento com maior feedback disponível para as correções no movimento poderia não ser sensível apenas às restrições do ID. Ademais, cada erro terminal no movimento induz uma perturbação imposta a condições iniciais do próximo movimento, o que poderia magnificar o erro na medida em que o número de tentativas movimentos é aumentado (CROSSMAN; GOODEVE, 1963/I983; MEYER et al., 1982). Assim, uma associação TM $\times$ ID diferente da proposta pela lei de Fitts seria esperada. Por outro lado, se considerada a explicação de Fitts, na qual existe uma capacidade única e limitada de processamento de informação, quando mantidas constantes as restrições espaciais que atuam sobre o movimento, o movimento não seria sensível à manipulação do número de tentativas (exceto se resultar em fadiga). Desta forma, a análise da manipulação no número de tentativas da tarefa de contornar figuras geométricas pode auxiliar no entendimento das estratégias de controle utilizadas na regulação da relação inversa velocidade-precisão.

O presente estudo analisou a relação $T M \times I D$, em tarefa de restrição espacial simulada por computador, na qual figuras geométricas foram manipuladas através de suas dimensões (perímetro), tamanho da linha (espessura), número de voltas para contornar a figura e forma (triângulo, quadrado e diamante).

\section{MÉTODOS}

\section{AMOSTRA}

Participaram do estudo dez voluntários (3 homens e 7 mulheres) com idade média de 24,8 anos (DP $=1,7$ ). Foram selecionados participantes que reportassem 
possuir pelo menos um computador em casa e o utilizam mais do que 6 horas semanais. Antes do início da avaliação os participantes foram informados dos procedimentos da pesquisa e assinaram um termo de consentimento livre e esclarecido. O presente estudo foi aprovado pelo Comitê de Ética em Pesquisa na universidade local (Parecer n 215/I0, Protocolo n²2 I7I/I0, CAE n 0197.0.268.000-10).

\section{EQUIPAMENTO E TAREFA}

Um computador da marca Kennex, um monitor de tela com I 5,4 polegadas e um mouse óptico da marca Satellite foram utilizados no experimento. O software Draw Task vI.5 (OKAZAKI, 2007) permitiu a manipulação da forma, do tamanho e da espessura das linhas da figura, além de fornecer a medida do tempo de movimento na tarefa. A tarefa constituiu em contornar figuras geométricas com o cursor do mouse através de um computador. A tarefa iniciava quando o sujeito segurava o botão esquerdo do mouse sobre uma das extremidades do desenho e terminava quando o sujeito soltava o botão do mouse na mesma posição inicial demarcada após completar o número de voltas desejado.

\section{PROCEDIMENTOS EXPERIMENTAIS}

Os sujeitos foram posicionados sentados com braços apoiados, de maneira confortável, em frente a um computador com o monitor levemente abaixo do nível dos olhos. Os sujeitos foram orientados a manter o braço que realizou a tarefa relaxado, ou seja, apoiado no suporte da cadeira, entre as tentativas. Também poderiam ser solicitados intervalos entre as tentativas por parte dos sujeitos. Estes cuidados foram tomados com o intuito de evitar a fadiga durante a realização do experimento.

Três figuras foram utilizadas (quadrado, triângulo e diamante), nos quais foram manipulados os tamanhos da figura através do perímetro (5। 4, 77। , 1028 pixels) e do número de voltas para contornar a figura ( I , 2, 4 e 8 voltas) e a espessura da linha da figura (14, 28, 56 pixels). Assim, um total de 36 figuras com índices de dificuldade (ID) entre 2,32 e 8,46 bits foram utilizados. Os IDs foram calculados através da equação proposta originalmente por Fitts (1954), a saber: $\log _{2}(2 D / A)$, no qual $A$ (alvo) foi relativo ao tamanho da espessura da linha e $D$ (distância) foi calculado de duas formas, a partir do produto entre o perímetro e o número de voltas (ID) e apenas através do perímetro da figura (índice de dificuldade normalizado ou $I D_{\text {norm }}$ ). As sequências das figuras e suas características de espessura de linha, perímetro e número de voltas foram randomizadas entre as tentativas. 
Os sujeitos receberam primeiramente instruções verbais de como realizar a tarefa: ( I ) Coloque o cursor do mouse em cima da linha do desenho; (2) clique o botão esquerdo do mouse e contorne o desenho mantendo o botão pressionado; (3) quando completar o número de voltas desejado solte o botão; (4) realize o movimento o mais rápido possível sem deixar o cursor sair da linha do desenho; (5) depois, os sujeitos observaram uma demonstração visual realizada pelo avaliador para iniciar um período de adaptação com 5 tentativas. Ao final de cada tarefa o programa forneceu o tempo de duração/movimento na tarefa (TM). Cada participante realizou três tentativas em cada uma das 36 figuras geométricas, sendo que apenas o menor tempo de cada uma delas foi analisado. As tentativas em que as restrições espaciais não foram respeitadas, delimitadas pela espessura da linha (demanda de acurácia na tarefa), foram repetidas imediatamente após o erro ser cometido.

\section{ANÁLISE ESTATÍSTICA}

Para analisar a relação inversa velocidade-acurácia, através da formulação da lei de Fitts na tarefa proposta, foram selecionadas as seguintes variáveis: tempo de movimento (TM) e índice de dificuldade (ID) do movimento. Para tanto, foi utilizada uma análise de regressão linear simples e de regressão não linear exponencial, com o nível de significância estabelecido em $p<0,05$. Os testes estatísticos foram realizados através do software SPSS (v. I0.0).

\section{RESULTADOS}

As figuras I, 2 e 3, apresentaram as relações TM x ID para as figuras triângulo, círculo e quadrado. A figura I apresenta a relação $T M \times I D$, em função do número de voltas (I, 2, 4 e 8) realizadas para cada condição de movimento. A figura 2 apresenta as condições agrupadas do TM associado ao ID para analisar a melhor equação de predição (linear ou exponencial). A figura 3 apresentara a relação TM x ID, sem considerar o número de voltas para contornar a figura como restrição sobre o movimento. Ou seja, o TM foi normalizado pelo número de voltas (TM dividido pelo número de voltas) e considerando apenas as restrições do tamanho da figura e da espessura da linha $\left(I D_{\text {norm }}\right)$. 

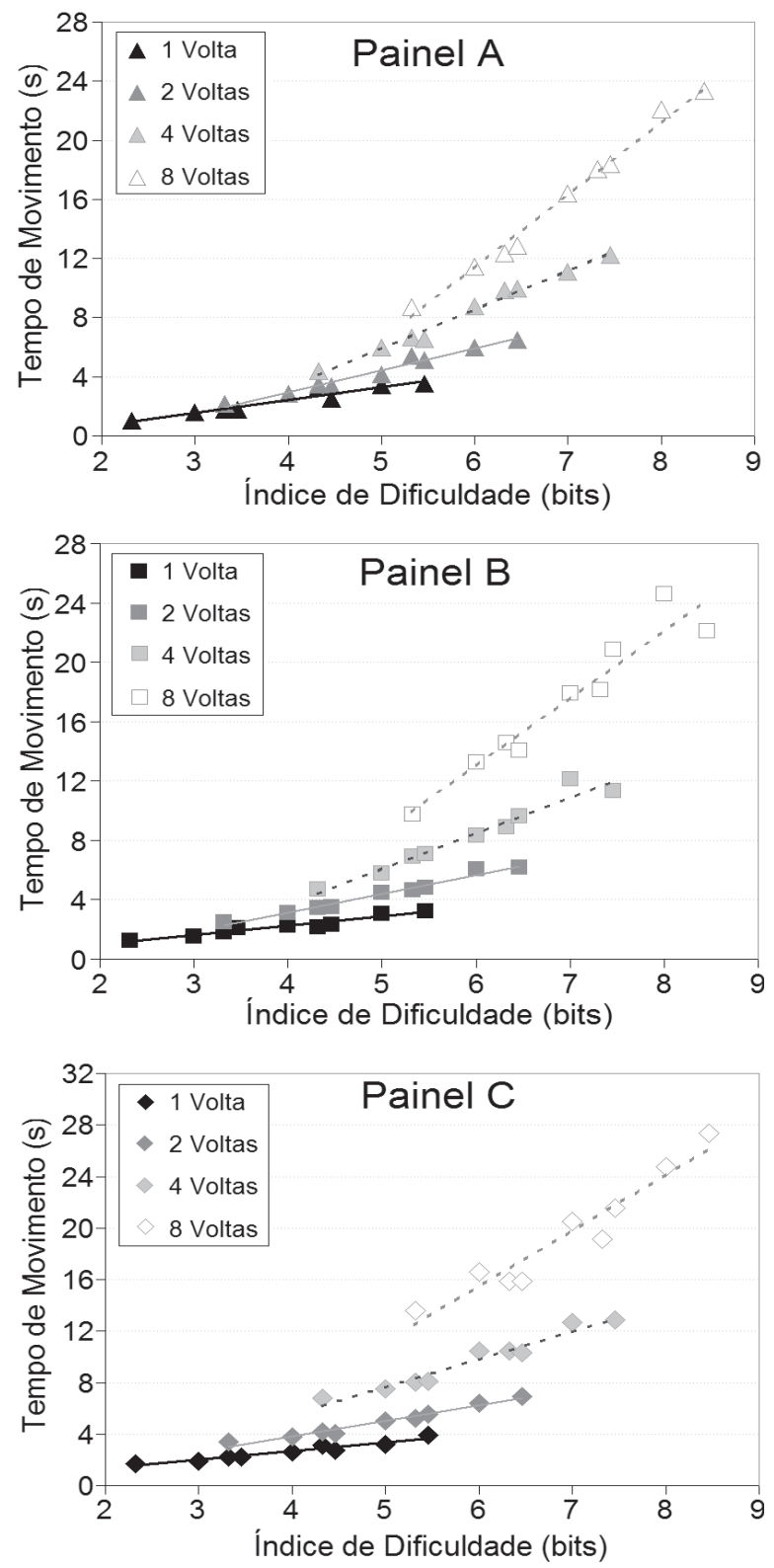

Figura I. Análise TM x ID (dados agrupados e separados em função do número de voltas) para as figuras do triângulo (Painel A), quadrado (Painel B) e diamante (Painel C), com retas de equação preditiva linear. 

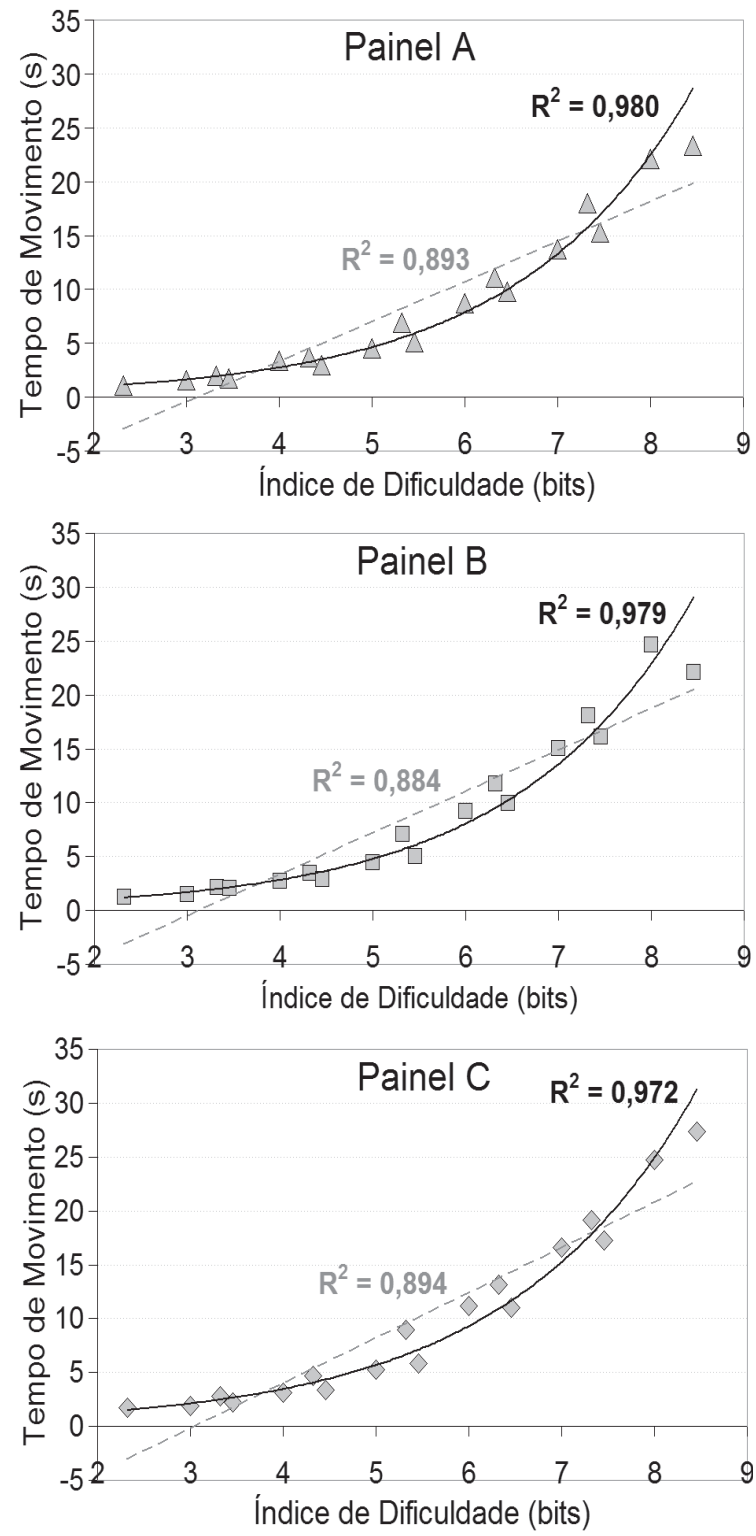

Figura 2. Função das retas e curvas de predição do comportamento da relação TM $\times$ ID para as figuras do triângulo (Painel A), quadrado (Painel B) e diamante (Painel A).

Legenda: $R^{2}$ representa o coeficiente de determinação como indicador do ajuste da reta e da curva de predição dos dados. 

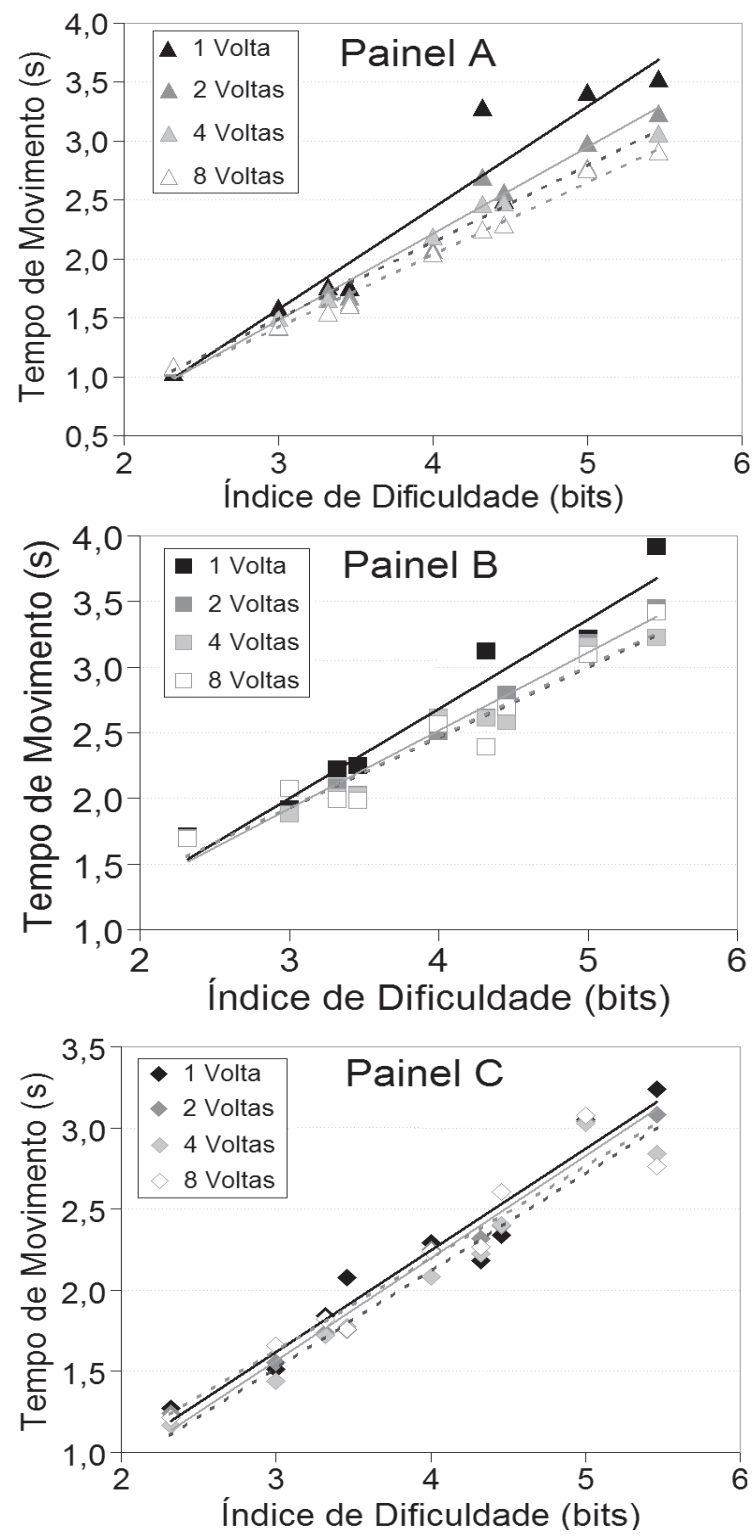

Figura 3. Análise TM x ID (dados agrupados, agrupados em função do número de voltas) para as figuras do triângulo (Painel A), quadrado (Painel B) e diamante Painel. 
Na tabela I foram expressos os coeficientes da Análise de Regressão Linear entre $T M \times I D$, para as três figuras. Como a normalização do TM modificou sua magnitude de forma homogênea os dados (pois todos são divididos por uma constante - número de voltas para contornar a figura), as Análises de Regressão Linear $T M \times I D$ e $T M \times I D_{\text {norm }}$, apresentaram os mesmos resultados. Por esta razão, tais análises serão apresentadas uma única vez por meio da relação TM x ID (Tabela I). Estas análises demonstraram que, independente da figura analisada, houve relação $T M \times I D$ com coeficientes de determinação $R^{2}>0,9\left(F_{1,7}>65,0 ; p<0,000 I\right)$. Estes resultados indicaram a forte associação entre $T M \times I D$ e $T M \times I D_{\text {norm }}$ na tarefa de contornar figuras geométricas.

Tabela I. Coeficientes da Análise de Regressão Linear entre os TM x ID nas figuras triângulo, quadrado e diamante.

\begin{tabular}{|c|c|c|c|c|c|}
\hline \multirow{2}{*}{$\frac{\text { Figura }}{\text { Triângulo }}$} & \multirow{2}{*}{$\frac{\text { Voltas }}{1}$} & \multicolumn{4}{|c|}{ Coeficientes da Análise de Regressão Linear TM × ID } \\
\hline & & $r=0,957$ & $R^{2}=0,916$ & $F_{1,7}=65,29$ & $p<0,0001$ \\
\hline Quadrado & I & $r=0,967$ & $R^{2}=0,94 I$ & $F_{1,7}=111,24$ & $p<0,0001$ \\
\hline Diamante & I & $r=0,969$ & $R^{2}=0,938$ & $F_{1,7}=106,5$ & $p<0,0001$ \\
\hline Triângulo & 2 & $r=0,987$ & $R^{2}=0,975$ & $F_{1,7}=271,32$ & $p<0,000$ I \\
\hline Quadrado & 2 & $r=0,987$ & $R^{2}=0,974$ & $F_{1,7}=265,38$ & $p<0,0001$ \\
\hline Diamante & 2 & $r=0,986$ & $R^{2}=0,95 I$ & $F_{1,7}=248,97$ & $p<0,000$ I \\
\hline Triângulo & 4 & $r=0,993$ & $R^{2}=0,986$ & $F_{1,7}=495,45$ & $p<0,0001$ \\
\hline Quadrado & 4 & $r=0,977$ & $R^{2}=0,954$ & $F_{1,7}=145,45$ & $p<0,0001$ \\
\hline Diamante & 4 & $r=0,975$ & $R^{2}=0,951$ & $F_{1,7}=136,46$ & $p<0,0001$ \\
\hline Triângulo & 8 & $r=0,994$ & $R^{2}=0,988$ & $F_{1,7}=595,50$ & $p<0,0001$ \\
\hline Quadrado & 8 & $r=0,959$ & $R^{2}=0,920$ & $F_{1,7}=81,18$ & $p<0,000$ \\
\hline Diamante & 8 & $r=0,962$ & $R^{2}=0,925$ & $F_{17}=86,94$ & $p<0,0001$ \\
\hline
\end{tabular}

A análise de regressão linear $T M \times I D$, considerando juntamente as condições de restrição de forma agrupada (tamanho da figura, espessura da linha e número de voltas), apresentou $R^{2}=0,813$ para o triângulo $\left(F_{1,34}=\mid 43,58\right.$; $p<0,000$ I; Painel $D), R^{2}=0,768\left(F_{1,34}=1\right.$ I2,90; $p<0,000$ I; Painel E) para o quadrado e $R^{2}=0,752$ $\left(F_{1,34}=103,35 ; p<0,0001\right.$; Painel $\left.F\right)$ para o diamante. Entretanto, as três figuras demonstraram melhores ajustes quando uma função exponencial foi utilizada para a predição TM $\times$ ID. Na qual, foi verificada uma associação exponencial TM $\times$ ID para o triângulo com $R^{2}=0,919\left(F_{1,34}=378,15 ; p<0,000\right.$ I; Painel $\left.D\right)$, para o quadrado 
com $R^{2}=0,877\left(F_{1,34}=242,09 ; p<0,000\right.$ I; Painel E) e para o diamante com $R^{2}=$ $0,838\left(F_{1,34}=378,15 ; p<0,000\right.$ I; Painel $\left.F\right)$. Tais resultados sugerem que, se considerado o número de voltas como parte da restrição imposta sobre a tarefa, a relação TM x ID é melhor explicada por uma função exponencial ao invés de linear.

\section{DISCUSSÃO}

A lei de Fitts (1954) é uma relação bem estabelecida que prediz o tempo de movimento através da especificação de alguns parâmetros espaciais da tarefa definindo um índice de dificuldade (ID). No qual, o tamanho do alvo (A) e a distância de movimento $(D)$ interagem $(I D=[2 D / A])$ para definir a quantidade de restrição imposta sobre o movimento em bits. De forma que, quanto maior à restrição imposta sobre o movimento, ou seja, grandes IDs, a tarefa seria desempenhada com maior tempo de movimento. Corroboração com esta predição do tempo de movimento por meio deste ID foi realizada em diversas tarefas, tais como: discretas (CARLTON, 1979; BARRETT; GLENCROSS, 1989; SMITS-ENGELSMAN et al., 2006), cíclicas (GUIARD, 1997; MOTTET et al., 200 I; SALMONI, 1983), de lançamentos (KERR; LANGOLF, 1977), de extensão de braço (KERR; LANGOLF, 1977), de flexão do braço (CORCOS et al., 1988), toques com ponteira (FITTS, 1954; FITTS; PETERSEN, 1964; OKAZAKI et al., 2008), de tal forma que esta formulação matemática tem sido um dos fenômenos mais consistentes nos estudos em comportamento motor (SPARROW; SPARROW, I 99। ; PLAMONDON; ALIMI, 1997; BOOTSMA et al., 2004).

Neste estudo, foi analisada a tarefa de contornar figuras geométricas. Para tanto, a restrição imposta sobre o movimento foi realizada através da manipulação de um ID determinado através da espessura da linha (relativo ao tamanho do alvo) e do perímetro a ser contornado de uma figura geométrica (relativo à distância de movimento). Por conseguinte, foi esperada a mesma predição de Fitts, na qual mudanças no ID estariam sistematicamente associadas com a duração do movimento. Tais suposições foram confirmadas nas análises individuais (sem o agrupamento do número de voltas; painéis do A até F), pois todas as figuras geométricas (triângulo, quadrado e diamante) demonstraram uma relação linear entre o TM e o ID na tarefa. Por conseguinte, a tarefa de contornar figuras geométricas simulada em computador também demonstrou suporte para as predições realizadas pela lei de Fitts. Tais resultados corroboram com a grande consistência deste paradigma, mesmo em tarefas mais complexas como a manipulação do uso de mouse em computador para restrições espaciais bidimensionais.

Outra restrição analisada, além das restrições espaciais de tamanho da figura e espessura da linha, foi o efeito do número de voltas para contornar a figura sobre 
a relação $T M \times I D$. Apesar dos estudos iniciais no paradigma velocidade-acurácia não contemplarem o efeito da repetição de um movimento sobre as estratégias de controle, têm sido demonstradas estratégias de controle particulares a esta restrição (GUIARD, 1993, 1997). Por exemplo, movimentos discretos permitem a especificação dos parâmetros de controle das posições iniciais e finais do sistema efetor antes mesmo de realizar a tarefa através de um sistema de controle via feedfoward (FITTS; PETERSEN, 1964). Por outro lado, em tarefas cíclicas, devido à especificação dos parâmetros de controle durante a realização do movimento, chamado de paralelismo parcial, cada erro terminal de movimento que se repete induz uma perturbação imposta a condições iniciais do próximo movimento, o que poderia magnificar o erro na medida em que o número de tentativas movimentos é aumentado (FITTS; PETERSEN, 1954; GUIARD, 1997). Como consequência, uma proporção de tempo de movimento na tarefa cíclica incluiria algum tempo extra para processar as informações de feedback (GUIARD, 1997). Em função destas particularidades, era esperado que o aumento no número de tentativas para contornar a figura, levasse a uma associação TM x ID diferente da proposta por Fitts ( 1954).

A relação $T M \times I D$, quando considerado o número de repetições da tarefa, apresentou uma associação mais forte através de uma função exponencial, ao invés de uma função linear (Painéis D, E e F). Pois, houve um aumento mais abrupto no TM nas condições de maior ID, principalmente, em função do maior número de voltas para contornar as figuras. Tais resultados poderiam sugerir que o número de voltas para contornar a figura teve um aumento exponencial, na relação $T M \times I D$, em função das correções via feedback no qual no qual um resíduo de erro entre as tentativas teria aumentado a demanda de tempo para as correções. Entretanto, a análise das associações $T M \times I D_{\text {norm }}$ (Painéis $\mathrm{G}, \mathrm{H}$ e I), ou seja, quando não foi considerado o número de voltas como restrição sobre o movimento e o tempo de movimento foi normalizado pelo número de voltas (dividido o TM total pelo número de voltas), não foram verificadas associações diferentes das predições de Fitts (FITTS, 1954; FITTS; PETERSEN, 1964). Por conseguinte, ainda que estratégias particulares de controle sejam utilizadas na regulação de movimentos cíclicos e discretos, quando mantidas constantes as restrições espaciais do movimento (distância de movimento e demanda de acurácia - tamanho do alvo) e o número de repetições da tarefa, a lei de Fitts foi robusta para explicar os dados. Todavia, quando o paradigma velocidadeacurácia for analisado também em função do número de repetição da tarefa (por exemplo: com movimentos discretos e cíclicos) o índice de dificuldade pode ser calculado a partir do número de tentativas, mas apresentando uma relação TM $x$ ID exponencial ao invés de logarítmica.

Em se tratando das características peculiares de cada desenho geométrico, poderiam ser esperados desempenhos particulares em função das restrições es- 
pecíficas fornecidas pelas figuras. Contudo, a análise descritiva e a associação TM $\times$ ID nas diferentes figuras sugeriram que essa particularidade não ocorreu. Assim, mesmo que o triângulo tenha ângulos mais agudos que o quadrado e o diamante, resultando em mudanças de direção mais abruptas no movimento, o triângulo possui menor número de vértices (3 vértices) quando comparado às outras duas figuras (4 vértices). Por conseguinte, pareceu haver uma compensação entre estas restrições (número de vértices e magnitude angular dos vértices), pois independente das características geométricas de cada figura os tempos de movimento foram muito próximos. Entretanto, mais estudos que manipulem estes fatores de restrição espacial (ângulo da figura e número de vértices - mudança de direção) são necessários para firmar tais suposições. Estes resultados sugerem que as maiores restrições sobre o desempenho na tarefa com restrição espacial estariam mais associadas à delimitação da espessura da linha (tamanho do alvo) e do perímetro da figura (distância de movimento), similar às predições da lei de Fitts demonstrando sua robustez.

O presente estudo analisou a tarefa de contornar figuras geométricas, atribuindo restrições espaciais ao movimento através da manipulação na espessura da linha (relativo ao tamanho do alvo), no perímetro da figura (relativo à distância de movimento) e no número de voltas para contornar a figura, para analisar o paradigma da relação inversa velocidade-acurácia. Foi verificada a robustez na lei de Fitts nesta classe de movimentos através de uma relação linear entre TM × ID quando não foi manipulado o número de voltas ao redor da figura. Contudo, quando considerado o número de voltas para contornar a figura na determinação do ID, à medida que este número de voltas foi aumentando, a relação TM × ID demonstrou melhor ajuste através de uma função exponencial, ao invés da linear originalmente proposta por Fitts (FITTS, 1954; FITTS; PETERSEN, 1964). Desta forma, foi sugerido que análises que contemplem o número de repetição da tarefa (por exemplo: com movimentos discretos e cíclicos) o índice de dificuldade pode ser calculado a partir do número de tentativas da tarefa, apresentando uma relação TM x ID exponencial.

\section{Speed-accuracy trade-off in task of drawing geometric figures}

ABSTRACT: The present study analyzed the speed-accuracy trade-off, through Fitts' law, in tasks of drawing geometric figures. A software specially developed allowed to manipulate the figure shape (square, triangle, and diamond) and size (perimeter and number of laps to contour around the figure), and line width, providing index of difficult (ID) between 2,32 and 8,46 bits. It was verified Fitts' law robustness on the task of drawing geometric figures. Furthermore, it was suggested that, in analysis dealing with the number of task repetition (such as discrete and cyclical movements), index of difficulty may be calculated by the number of trials, showing an exponential rather than linear TM $x$ ID association.

KEY WORDS: Speed; Accuracy; movement; motor control. 


\section{Relación velocidad-precisión en las tareas del dibujar cifras geométricas}

RESUMEN: Eso studio examinó el paradigma de la relacion inversa velocidad-precisión, por la lei de Fitts, en tareas de contornar figuras geométricas. Un software especialmente desarrollado permite manipular la forma (cuadrado, triángulo y el diamante) y el tamaño de la figura (el perímetro y el número de vueltas para llegar a su alrededor), y el tamaño de línea, proporcionando índices de dificuldad (ID) entre 2,32 y 8,46 bits. Se verificó la robustez de la ley de Fitts en la tarea de contornar figuras geométricas simulados en el computador. Además, fue sugerido que las análisis utilizando el número de repetición de la tarea (por ejemplo: con los movimientos discreto y cíclicos), el índice de dificuldad se puede calcularse por el número de intentos, mostrando una relación TM x ID exponencial en lugar de linear.

PALABRAS-CLAVE: Velocidad; precisión; movimiento; control motor.

\section{REFERÊNCIAS}

ANDRES, R. O.; HARTUNG, K. J. Prediction of head movement time using Fitts' law. Human Factors, New York, v. 31 , n. 6, p. 703-713, 1989.

BARRETT, N. C; GLENCROSS, D.J . Response amendments during manual aiming movements to double-step targets. Acta Psychologica, Oxford, v. 70, p. 205-217, 1989.

BEGGS W. D. A.; HOWART, C. I. The accuracy of aiming at a target: Some further evidence for a theory of intermittent control. Acta Psychologica, Oxford, v. 36, p. 17I$177,1972$.

BOOTSMA, R. J. et al.The speed-accuracy trade-off in manual prehension: Effects of movement amplitude, object size and object width on kinematic characteristics. Experimental Brain Research, Berlin, v.98, p. 535-54I, 1994.

BOOTSMA, R. J.; FERNANDEZ, L.; MOTTET, D. Behind Fitts' law: kinematic patterns in goal-directed movements. International Journa of Human-Computer Studies, New York: Academic Press, v. 61, n. 6, p. 81 I-821, 2004.

CARLTON, L. G. Control processes in the production of discrete aiming responses. Journal of Human Movement Studies, London, v. 5, p. I I 5- 1 24, 1979.

CORCOS, D. M.; GOTTLIEB, G. L.; AGARWAL, G. C. Accuracy constraints upon rapid elbow movements. Journal of Motor Behavior, Washington, v. 20, n. 3, p.255-72, 1988.

CROSSMAN, E. R. F. W.; GOODEVE, P. J. Feedback control of hand movement and Fitts' law. Quarterly Journal of Experimental Psychology, Cambridge, v. 35A, p. 25I-278, 1983.

FITTS, P. M. The information capacity of the human motor system in controlling the amplitude of movement. Journal of Experimental Psychology: General, Washington, v. 3, p. 262-269, 1954. 
FITTS, P. M.; PETERSON, J. R.Information capacity of discrete motor responses. Journal of Experimental Psychology: Learning, Memory and Cognition, Washington, v. 67, p. I03I 12, 1964.

GUIARD, Y. Fitts' law in the discrete vs. cyclical paradigm. Human Movement Science, Amsterdam, v.16, p. 97-131, 1997.

HUYS, R. et al. Fitts' law is not continuous in reciprocal aiming. Proceedings The Royall Society Biollogical Sciences, vol. 277, n. I 179- I I 84, 2010.

KERR, B. A.; LANGOLF, G. D. Speed of aiming movements. Quarterly Journal of Experimental Psychology, Cambridge, v. 29, p. 475-481, 1977.

MILNER, G. A. What is information measurement? American Psychologist, v.8, p. 3- I I, 1953.

MEYER, D. E. et al. Optimality in Human Motor Performance: Ideal Control of Rapid Aimed Movements. Psychological Review, Washington, v. 95, n.3, p. 340-370, 1988.

MEYER, D. E.; SMITH, J. E. K, WRIGHT, C. E. Models for the Speed and Accuracy of Aimed Movements. Psychological Review, Washington, v. 89, n. 5, p. 449-482, 1982.

MOTTET, D. et al. Two-handed performance of a rhythmical Fitts task by individuals and dyads. Journal of Experimental Psycology: Human Perception an Performance, Washington, v. 6, p. |275-|286, 200|.

OKAZAKI, V. H. A. Software que analisa a tarefa de contornar figuras geométricas em computador. Draw Task (v. I.5). 2007. Disponível em <http://okazaki.webs.com>. Acesso em: 04 set. 2009.

OKAZAKI, V. H. A. et al. Modelo estocástico de sub-movimentos otimizados em Movimentos com restrição espacial simulados em Computador. Revista Brasileira de Biomecânica, São Paulo, Ano 9, n. 16, 2008.

PLAMONDON, R.; ALIMI, A. M. Speed/accuracy trade-offs in target-directed movements. Behavior and Brain Scienses, Cambridge v. 20, p. 279-249, 1997.

SALMONI, A. W. A descriptive analysis of children performing Fitts' reciprocal tapping task. Journal of Human Movement Studies, London, v. 9, p. 8I- 195, 1983.

SHANNON, C. E. A mathematical theory of communication. Bell System Technical Journal, v.27, p.623-656, 1948.

SLIFKIN, A. B.; GRILLI, S. M. Aiming for the future: prospective action difficulty, prescribed difficulty, and Fitts' law. Experimental Brain Research, Berlin, v. I74, p. 746-753, 2006.

SMITS-ENGELSMAN, B. C. M.; SWINNEN, S. P.; DUYSENS, J. The advantage of cyclic over discrete movements remains evident following changes in load and amplitude. Neuroscience Letters, Amsterdam, v. 396, n. I, p. 28-32, 2006. 
SPARROW, W. A.; SPARROW, H. T. Trends in Motor Behavior Research: A Study Based on Citation Analysis. Journal of Human Movement Studies, v.21, p. 183-199, 1991.

WOODWORTH, R. S. The accuracy of voluntary movement. Psychological Review, Washington, Monografia Supplement, v. 3, p. I-1 19, 1899.

WU, C. C.; KWON, O. S.; KOWLER, E. Fitts's Law and speed/accuracy trade-offs during sequences of saccades: Implications for strategies of saccadic planning. Vision Research, v. 50, n. 21 , p. $2142-2157,2010$.

Recebido: 25 jan. 2010 Aprovado: 16 dez. 2010

Endereço para correspondência: Prof. Dr. Victor Hugo Alves Okazaki Departamento de Educação Física UEL Campus Universitário - Rodovia Celso Garcia Cid - Km 380 Londrina - PR - Brasil CEP: 8605 I-990 / Caixa Postal: 600 I 